\title{
Effects of a moving $X$-line in a time-dependent reconnection model
}

\author{
S. A. Kiehas ${ }^{1,2}$, V. S. Semenov ${ }^{3}$, I. V. Kubyshkin ${ }^{3}$, Yu. V. Tolstykh ${ }^{3}$, T. Penz ${ }^{1,2, *}$, and H. K. Biernat ${ }^{1,2}$ \\ ${ }^{1}$ Space Research Institute, Austrian Academy of Sciences, Schmiedlstrasse 6, 8042 Graz, Austria \\ ${ }^{2}$ Institute of Physics, University of Graz, Universitätsplatz 5, 8010 Graz, Austria \\ ${ }^{3}$ Institute of Physics, State University, St. Petersburg, 198504 Russia \\ *now at: INAF Osservatorio Astronomico di Palermo, Piazza del Parlamento 1, Palermo, Italy
}

Received: 4 August 2006 - Revised: 2 January 2007 - Accepted: 22 January 2007 - Published: 1 February 2007

\begin{abstract}
In the frame of magnetized plasmas, reconnection appears as an essential process for the description of plasma acceleration and changing magnetic field topology. Under the variety of reconnection regions in our solar system, we focus our research onto the Earth's magnetotail. Under certain conditions a Near Earth Neutral Line (NENL) is free to evolve in the current sheet of the magnetotail. Reconnection in this region leads to the formation of Earth- and tailward propagating plasma bulges, which can be detected by the Cluster or Geotail spacecraft. Observations give rise to the assumption that the evolved reconnection line does not provide a steady state behavior, but is propagating towards the tail (e.g., Baker et al., 2002). Based on a time-dependent variant of the Petschek model of magnetic reconnection, we present a method that includes an X-line motion and discuss the effects of such a motion. We focus our main interest on the shock structure and the magnetic field behavior, both for the switch-on and the switch-off phase.
\end{abstract}

Keywords. Magnetospheric physics (Magnetotail; Storms and substorms) - Space plasma physics (Magnetic reconnection)

\section{Introduction}

Magnetic reconnection is an important energy converting plasma process, occurring at the solar corona or at planetary magnetospheres, for instance. Due to the interaction of two magnetized plasmas with opposite directed magnetic fields, initially separated by a current sheet, magnetic energy may be converted into kinetic energy of the plasma. Another important feature of this mechanism is the change of the magnetic field configuration, meaning that magnetic field lines from initially different topological regions get merged.

Correspondence to: S. A. Kiehas

(stefan.kiehas@stud.uni-graz.at)
The dissipative processes, leading to a field line merging in the so-called diffusion region, can be specified in terms of a source function $\mathbf{E}_{r}(t)$, the reconnection electric field (Biernat et al., 1987). This electric field appears as a result of a local breakdown of the ideal MHD constraint of infinite conductivity, meaning the appearance of a locally enhanced electric resistivity. With the release of stored magnetic field energy, incoming plasma gets accelerated and propagates along the current sheet with Alfvén velocity $v_{A}$. Decades ago, Petschek (1964) suggested that the appearance of a reconnection site is accompanied with the formation of large amplitude MHD waves and shocks that are generated inside the diffusion region (Heyn et al., 1988).

This steady-state model of standing waves was extended to a time-dependent model (e.g., Pudovkin and Semenov, 1985; Biernat et al., 1987; Rijnbeek et al., 1991; Semenov et al., 1992), including the basic Petschek mechanism. The time-dependent model acts on the assumption that enhanced electric resistivity appears in a local area in the current sheet, exhibiting the onset of magnetic reconnection. This active phase of reconnection is called the switch-on phase. Eventually, the reconnection electric field, generated by enhanced resistivity, drops to zero and reconnection ceases. At this moment, the switch-on phase passes into the switch-off phase. The shocks, previously generated in the diffusion region, detach from the reconnection site and propagate in opposite directions along the current sheet, enclosing the outflowing, accelerated plasma. For this reason, the area bounded by the shocks is called outflow region. An important feature is the fact that magnetic field lines from either side of the current sheet are connected via the shocks, as shown in Fig. 1. This implies the appearance of a topologically new region of reconnected flux, called field reversal region (FRR). The point, where the magnetic fields appear in an X-type configuration is called $\mathrm{X}$-point. In the three dimensional case we work with an X-line.

Published by Copernicus GmbH on behalf of the European Geosciences Union. 


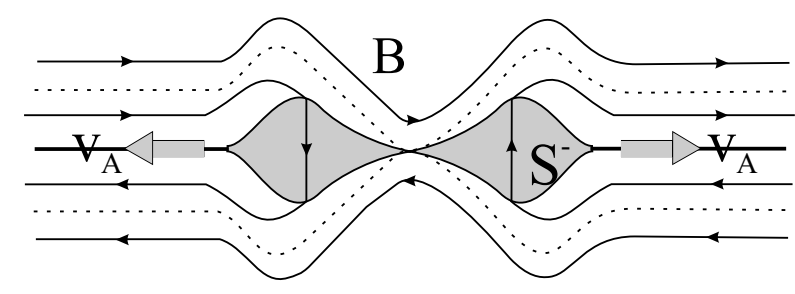

Fig. 1. Geometrical configuration of time-dependent reconnection. Two antiparallel directed magnetic fields are separated by a current sheet. The shocks, denoted by $S^{-}$, propagate in opposite directions along the current sheet. The shaded regions, enclosed by the shocks, represent the outflow or field reversal regions. The dashed lines denote the separatrices, which separate regions of different magnetic field topology (after Semenov et al., 2004).

Especially with the four Cluster spacecraft, exhibiting a tetrahedron constellation, it is possible to observe magnetic reconnection in Earth's magnetotail in more detail (e.g., Cattell et al., 2005). With this special spacecraft constellation it is possible to distinguish between temporal and spatial phenomena. One result of observing magnetic reconnection in the magnetotail was the indication of a tailward moving Xline (e.g., Runov et al., 2003). In the following, a model for time-dependent reconnection with a moving X-line is presented. Owen and Cowley (1987) showed that a moving $\mathrm{X}$-line leads to a compression of the magnetic field ahead the $\mathrm{X}$-line and, therefore, to an increase of $B_{z}$ as well as a thickening of the field and particle layers. Behind the X-line, a decrease of $B_{z}$ and thinner layers occur. Whereas Owen and Cowley (1987) implemented a sudden change in the reconnection rate in their model, the presented model is based upon a continuous build-up and decay of the reconnection rate, associated with impulsive reconnection.

In Sect. 2 we summarize the basic considerations, which are necessary for our investigations. In Sect. 3 we discuss the structure of the Petschek-shocks for the assumption of a moving X-line. Sections 4 and 5 deal with the behavior of the magnetic field in the outflow and inflow region, respectively. Effects of compressibility are discussed in Sect. 6. In Sect. 7 we show some qualitative observational aspects for spacecraft measurements in the vicinity of an reconnection event. A summary of our results is given in Sect. 8 .

\section{Basic considerations}

We consider two oppositely oriented magnetic fields of the same field strength, embedded in two identical, uniform and initially stationary incompressible plasmas, separated by a current sheet, modelled as a tangential discontinuity along the $\mathrm{x}$-axis. In our geometry the $\mathrm{x}$-axis is directed parallel and the $\mathrm{z}$-axis perpendicular to the current sheet. The magnetic field $\mathbf{B}$, total pressure $P$, composed of the plasma pressure $p$ and magnetic pressure $B^{2} / 8 \pi$, can be written as,

$\mathbf{B}_{1,2}=\left(B_{1,2}, 0\right)$,

$P_{1}=P_{2} \Rightarrow p_{1}+\frac{B_{1}^{2}}{8 \pi}=p_{2}+\frac{B_{2}^{2}}{8 \pi}$,

where subscripts 1 and 2 denote the upper and lower half plane, respectively. The density $\rho$, pressure $p$, magnetic field strength $B$ and plasma velocity $v$ are linked as

$\rho_{1}=\rho_{2}=$ const., $p_{1}=p_{2}$,

$B_{1}=-B_{2}=B_{0}$.

As a boundary condition, we assume a reconnection electric field $\mathbf{E}_{r}(t)$, acting as initiator of the reconnection process. With the inequality $E_{r} \ll E_{A}=B_{0} v_{A} / c$ we discuss this problem under the aspect of weak reconnection. This implies the identification of a small parameter

$\epsilon=\frac{E_{r}}{E_{A}} \ll 1$.

Quantity $E_{A}$ denotes the Alfvén electric field, formed by the initial magnetic field $B_{0}$ and the Alfvén velocity $v_{A}$. Considering active reconnection during the time interval $0<t \leq 1$, $E_{r}$ is modelled as a sine-pulse in the form,

$E_{r}(t)= \begin{cases}0 & t>1 \\ \sin (\pi t) & 0<t \leq 1 .\end{cases}$

By introducing the eight-dimensional MHD state vector (Rijnbeek and Semenov, 1993), information about all eight variables in the MHD equation set (e.g., Akhiezer et al., 1975) are summarized in one single quantity

$\mathbf{U}=\mathbf{U}(\rho, p, \mathbf{B}, \mathbf{v})$

Since we discuss weak reconnection, the small parameter $\epsilon$ allows us to expand the MHD variables by using an asymptotic series (see also Kiendl et al., 1997; Alexeev et al., 2001),

$\mathbf{U}=\mathbf{U}^{(0)}+\epsilon \mathbf{U}^{(1)}+\ldots$,

where subscripts 0 and 1 denote undisturbed and disturbed quantities, respectively. In the weak reconnection approximation, the outflow region can be considered as a boundary layer, meaning that the longitudinal scale in $\mathrm{x}$-direction is much larger than the perpendicular scale in z-direction (Sonnerup, 1970). An order-of-magnitude estimation gives (Semenov et al., 2004),

$P, v_{x}, B_{x}, x \sim 1$,

$v_{z}, B_{z}, z \sim \epsilon$.

Due to this approximation, we introduce new boundary layer variables,

$\tilde{x}=x, \tilde{B}_{x}=B_{x}, \tilde{v}_{x}=v_{x}, \tilde{P}=P$,

$\tilde{z}=z / \epsilon, \tilde{B}_{z}=B_{z} / \epsilon, \tilde{v}_{z}=v_{z} / \epsilon$. 
The behavior of the magnetic field and the plasma in the field reversal region can be determined by using the RankineHugoniot jump equations (e.g., Baumjohann and Treumann, 1996) and the equations of ideal MHD (e.g., Akhiezer et al., 1975). For the plasma velocity and the magnetic field we can write

$\tilde{v}_{x}^{(0)}(x, z, t)=v_{A}=$ const.

$\tilde{v}_{z}^{(1)}(x, z, t)=0$,

$\tilde{B}_{x}^{(0)}(x, z, t)=0$,

$\tilde{B}_{z}^{(1)}(x, z, t)=B_{0} g\left(x \mp v_{A} t\right)$,

as shown by Semenov et al. (2004) and Biernat et al. (1987). The function $g\left(x \mp v_{A} t\right)$ is an arbitrary function of its argument.

\section{Structure of the shock}

Inside the diffusion region so-called Petschek shocks are generated and propagate into the surrounding medium. We denote the shape of the shock by

$z=\epsilon f(x, t)$,

with the surface normal vector $\mathbf{n}=(\epsilon \partial f / \partial x,-1)$. The shock speed is given by $\mathbf{u}=(\partial x / \partial t, \partial z / \partial t)$. Thus, the corresponding shock speed normal to the surface can be written as $u=-\epsilon \partial f / \partial t$.

With the results of Biernat et al. (1987) we can write the function $f(x, t)$ from Eq. (3) in the upper half-plane

$f^{ \pm}(x, t)= \pm \frac{c}{v_{A} B_{0}} \frac{1}{\left(1 \mp \frac{U}{v_{A}}\right)^{2}}(x-U t) E_{r}\left(\frac{x \mp v_{A} t}{U \mp v_{A}}\right)$,

with $c$ and $U$ as the speed of light and the velocity of the reconnection line, respectively. Formulas are written in the CGS-unit system. Since we suppose an X-line motion with a constant velocity $U$ along the current sheet, it is necessary to distinguish between $x>U t$ and $x<U t$, denoted by plus and minus signs, respectively. The term $E_{r}\left(\left[x \mp v_{A} t\right] /\left[U \mp v_{A}\right]\right)$ represents the reconnection electric field as a function of its argument. For the special case $U=0$, the functions $f^{+}$and $f^{-}$are

$f^{+}(x, t)=f^{-}(-x, t)=\frac{c}{v_{A} B_{0}} x E_{r}\left(t-\frac{x}{v_{A}}\right)$,

which can also be found for the normalized case in the paper of Semenov et al. (2004). For the case $U=0$, the shape of the shock structures moving in positive and negative $\mathrm{x}$-direction is symmetric with respect to the z-axis, as shown in Fig. 2.

Since the switch-on phase lasts from $0<t \leq 1$, both panels show the shock structure during active reconnection. In the course of time, the structure blows up in $\mathrm{x}$ - and $\mathrm{z}$-direction,
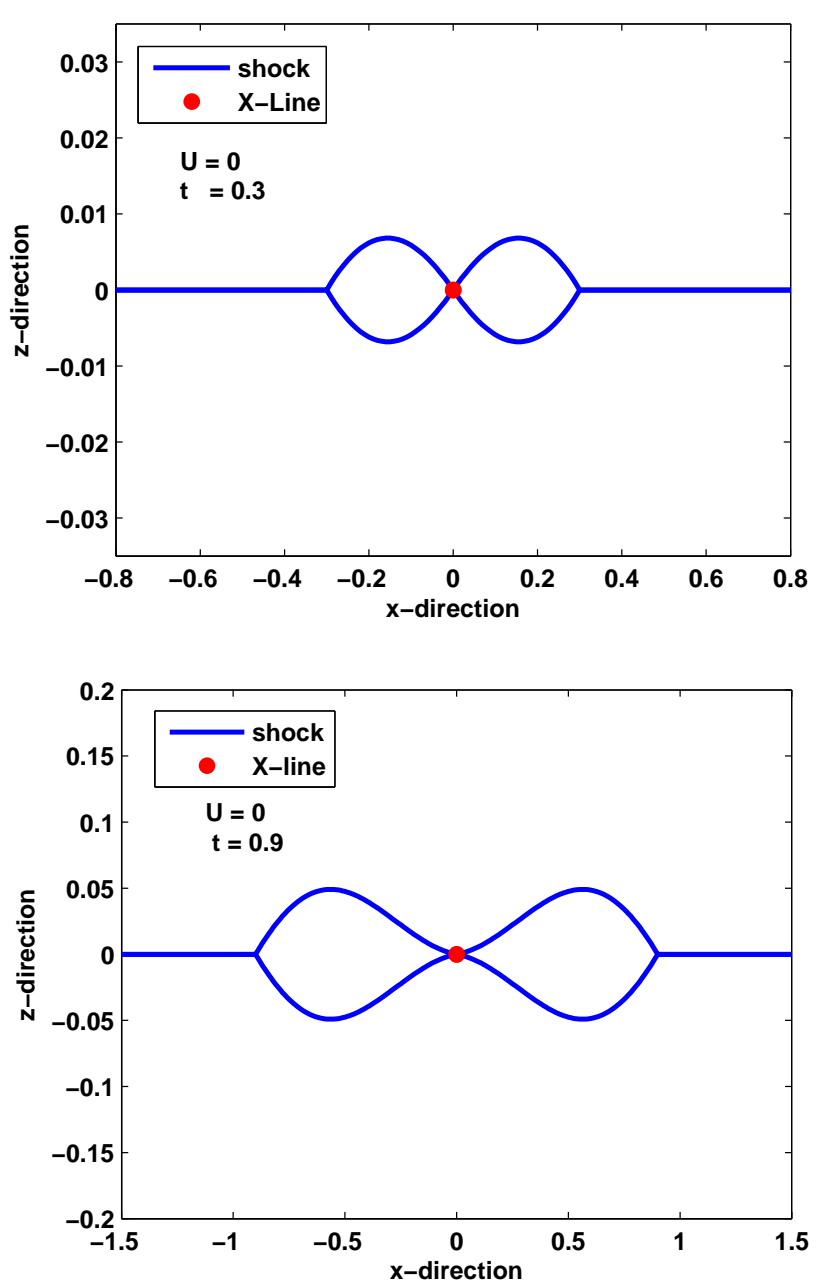

Fig. 2. Structure of the field reversal region during active reconnection (switch-on phase) for the times $t=0.3$ (upper panel) and $t=0.9$ (lower panel) and velocity of the reconnection line $U=0$. The reconnection line is denoted by a dot in the center, constant at $x_{r}=z_{r}=0$. Mind the different axis scaling in the upper and lower panel.

but still shows a symmetric behavior. This changing in shape and size can be seen in more detail in Fig. 3 .

Figure 4 shows the same situation for a moving reconnection line with a velocity $U=0.5 v_{A}$. The reconnection line, again denoted by a dot, reaches its maximum distance from the initial reconnection site $x=0.5$ when reconnection ceases. During this motion, the rightward evolved shocks get squeezed in $\mathrm{x}$-direction, whereas the leftward evolved shocks get stretched in $\mathrm{x}$-direction. For the z-elongation of the shocks the situation is vice versa.

Figure 5 shows the situation after reconnection ceased for different velocities of the reconnection line. Reconnection starts at time $t=0$, when in the diffusion region non-ideal MHD holds and finite conductivity appears. After time $t=1$, when the system returns into a stable state, the reconnection 


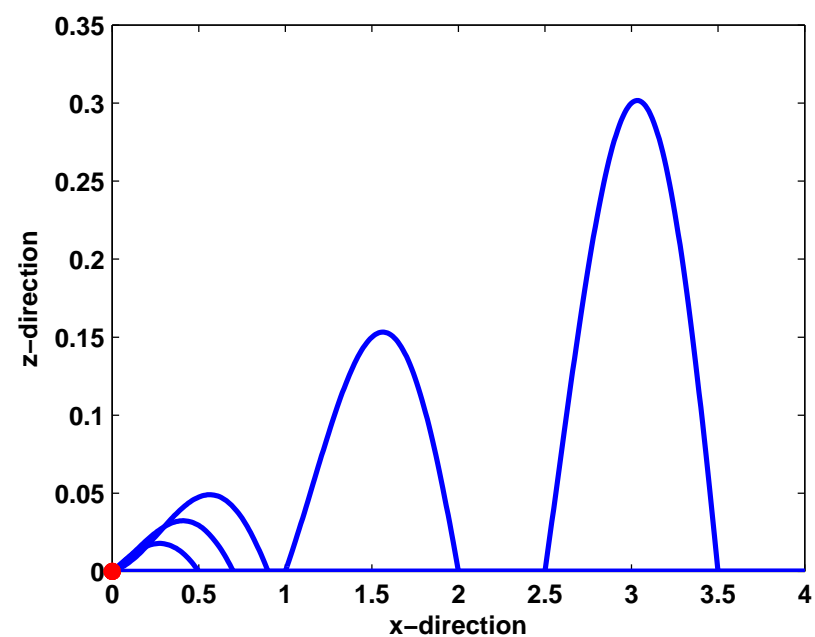

Fig. 3. Evolution of the shock structure in the first quadrant. For $U=0$ the shape of the shock is plotted for $t=0.5, t=0.7, t=0.9$, $t=2, t=3.5$ (from left to right).

electric field drops to zero. Up to the time of switch-off, the diffusion region can be considered as a continuous emitter of MHD waves. After switch-off no more MHD waves are generated by the reconnection process and no more reconnected flux is added to the system. This implies that the outflow regions detach from the original site of reconnection and the current sheet is re-established in its wake. Since the previously generated MHD waves continue to propagate, the outflow regions now appear as a pair of solitary waves propagating in opposite directions along the current sheet. In the course of time, the outflow regions continue to change both, in shape and size, as in the case for a fixed X-line.

With the lower panels of Fig. 5, a clear correlation between the X-line velocity and the degree of asymmetry between both shock structures is evident.

During the switch-on phase, the volume of the shocks increases nonlinearly, as it is shown in the upper panel of Fig. 6, which displays the growth of the shock during active reconnection during the time $0<t \leq 1$. After reconnection ceased, the growth increases linearly with time, as figured in the lower panel. There is no difference neither in total growth nor in the evolution between leftward and rightward propagating shocks. The values on the axis of ordinates in Fig. 6 do not represent true values for the volume of the shock. In the strict sense, the calculation of the volume is only the calculation of the surface area in the x-z-plane. Since no change in shape in y-direction is assumed, this function represents the temporal behavior of the shock structure.

\section{Magnetic field behavior in the outflow region}

For the computational analysis of the problem it is convenient to introduce normalized quantities. The normaliza-
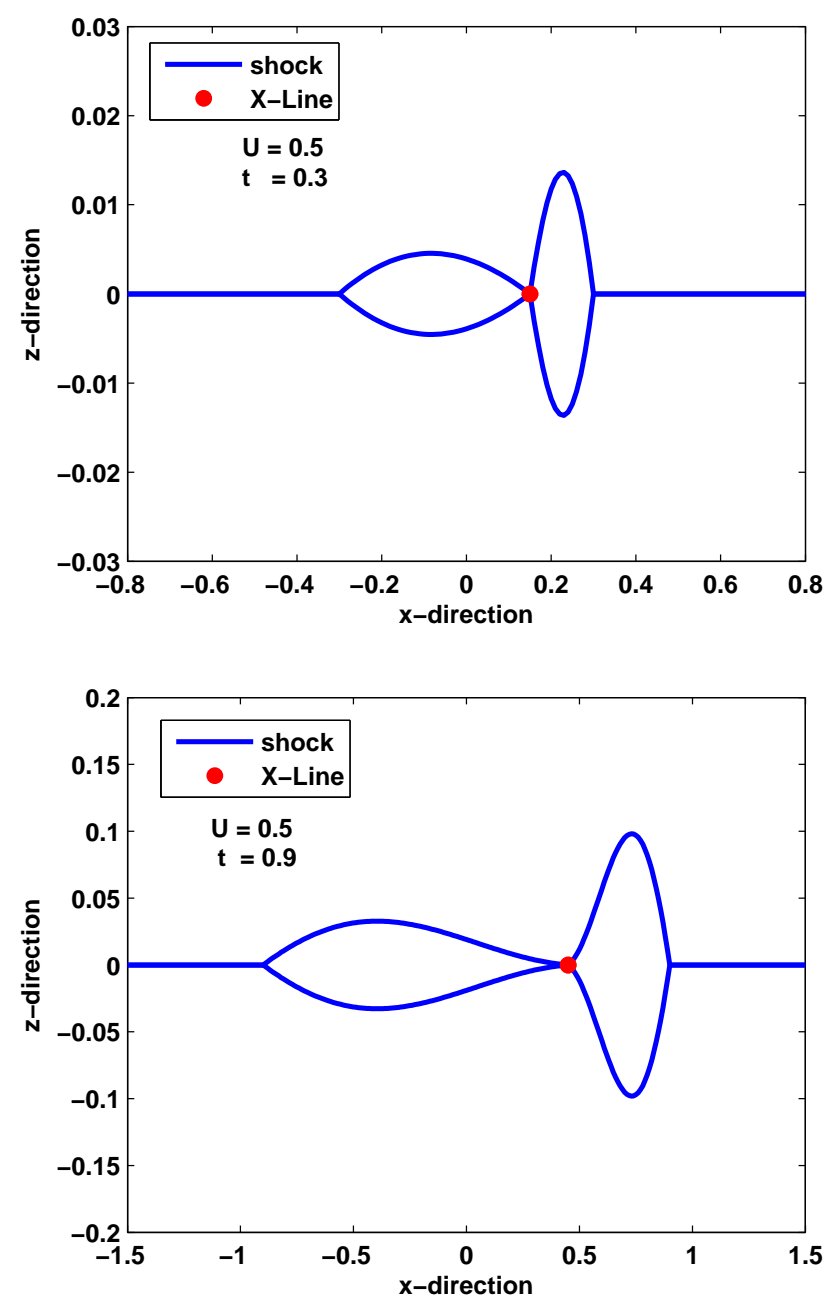

Fig. 4. Structure of the field reversal region during active reconnection (switch-on phase) for the times $t=0.3$ (upper panel) and $t=0.9$ (lower panel) and velocity of the reconnection line $U=0.5 v_{A}$. Mind the different axis scaling in the upper and lower panel.

tion is done with respect to the initial undisturbed magnetic field $B_{0}$ and the Alfvén velocity $v_{A}$. The component $\tilde{B}_{z}^{(0)}$ can be derived from the ideal MHD equations. Assuming $\tilde{\mathbf{v}}^{(0)}=( \pm 1,0)$ and $\tilde{B}_{x}^{(0)}=0$, this yields

$\frac{\partial \tilde{B}_{z}^{(0)}}{\partial t} \pm \frac{\partial \tilde{B}_{z}^{(0)}}{\partial x}=0$,

with $\tilde{B}_{z}^{(0)}=F((x \mp t) /(U \mp 1))$ as the general solution for this equation (Semenov et al., 2004). The arbitrary function $F((x \mp t) /(U \mp 1))$ can be concretized through the boundary condition at the reconnection line at $x=0$ by using the general form of the reconnection electric field for the normalized case, in the frame of reference of the reconnection line

$$
E_{r}=\left( \pm \tilde{v}_{x}^{(0)}-U\right) \tilde{B}_{z}^{(0)} .
$$



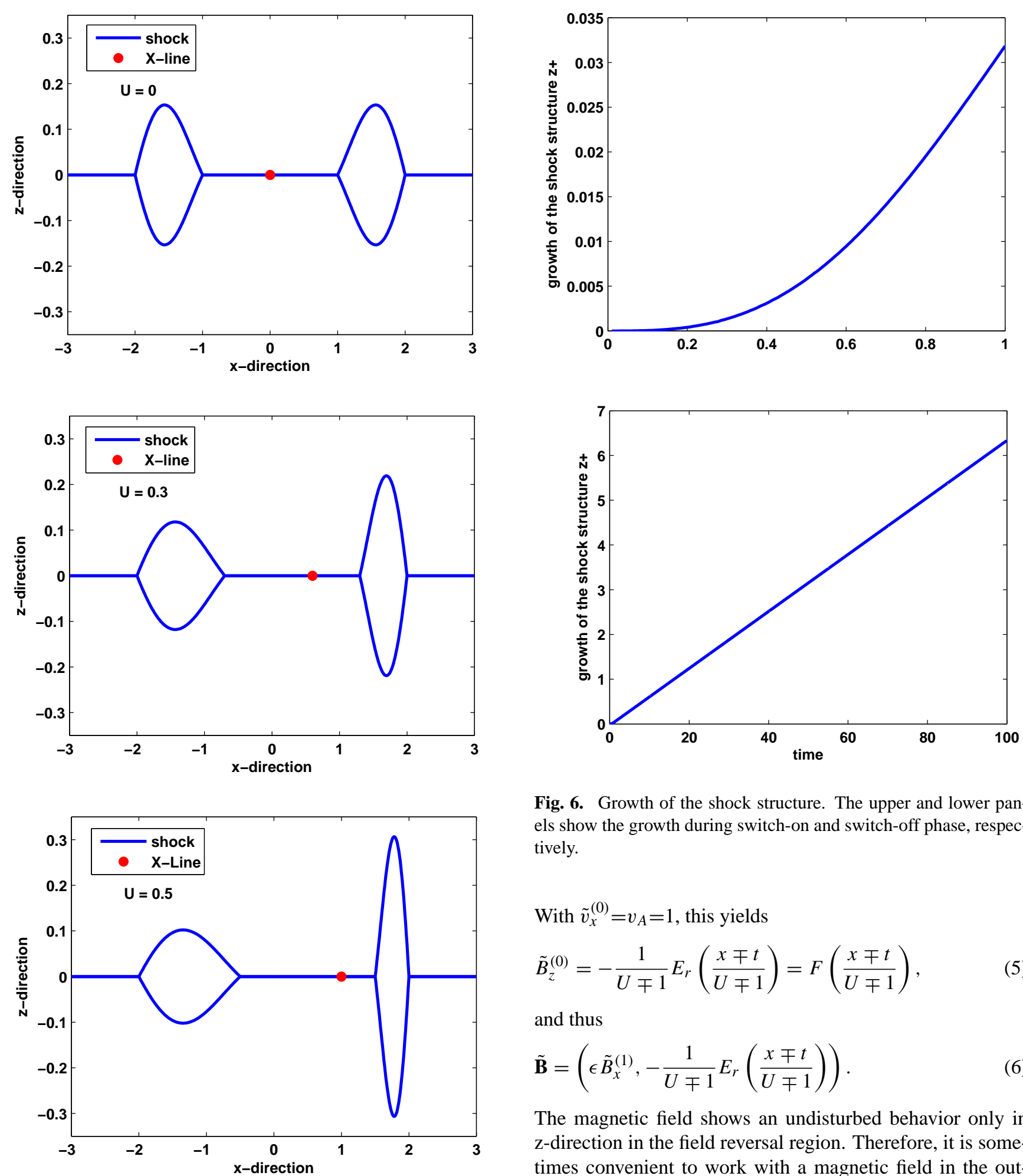

Fig. 6. Growth of the shock structure. The upper and lower panels show the growth during switch-on and switch-off phase, respectively.

With $\tilde{v}_{x}^{(0)}=v_{A}=1$, this yields

$\tilde{B}_{z}^{(0)}=-\frac{1}{U \mp 1} E_{r}\left(\frac{x \mp t}{U \mp 1}\right)=F\left(\frac{x \mp t}{U \mp 1}\right)$,

and thus

$\tilde{\mathbf{B}}=\left(\epsilon \tilde{B}_{x}^{(1)},-\frac{1}{U \mp 1} E_{r}\left(\frac{x \mp t}{U \mp 1}\right)\right)$.

The magnetic field shows an undisturbed behavior only in $\mathrm{z}$-direction in the field reversal region. Therefore, it is sometimes convenient to work with a magnetic field in the outflow region which has only a z-component. The behavior of the z-component of the magnetic field is shown in Fig. 7.

Fig. 5. Shape of the shocks. The upper panel shows the shape of the shock structures for the case $U=0$ and $t=2$, the middle for $U=0.3 v_{A}$ and $t=2$ and the lower panel for the case $U=0.5 v_{A}$ and $t=2$. The dot represents the final position of the reconnection line. 

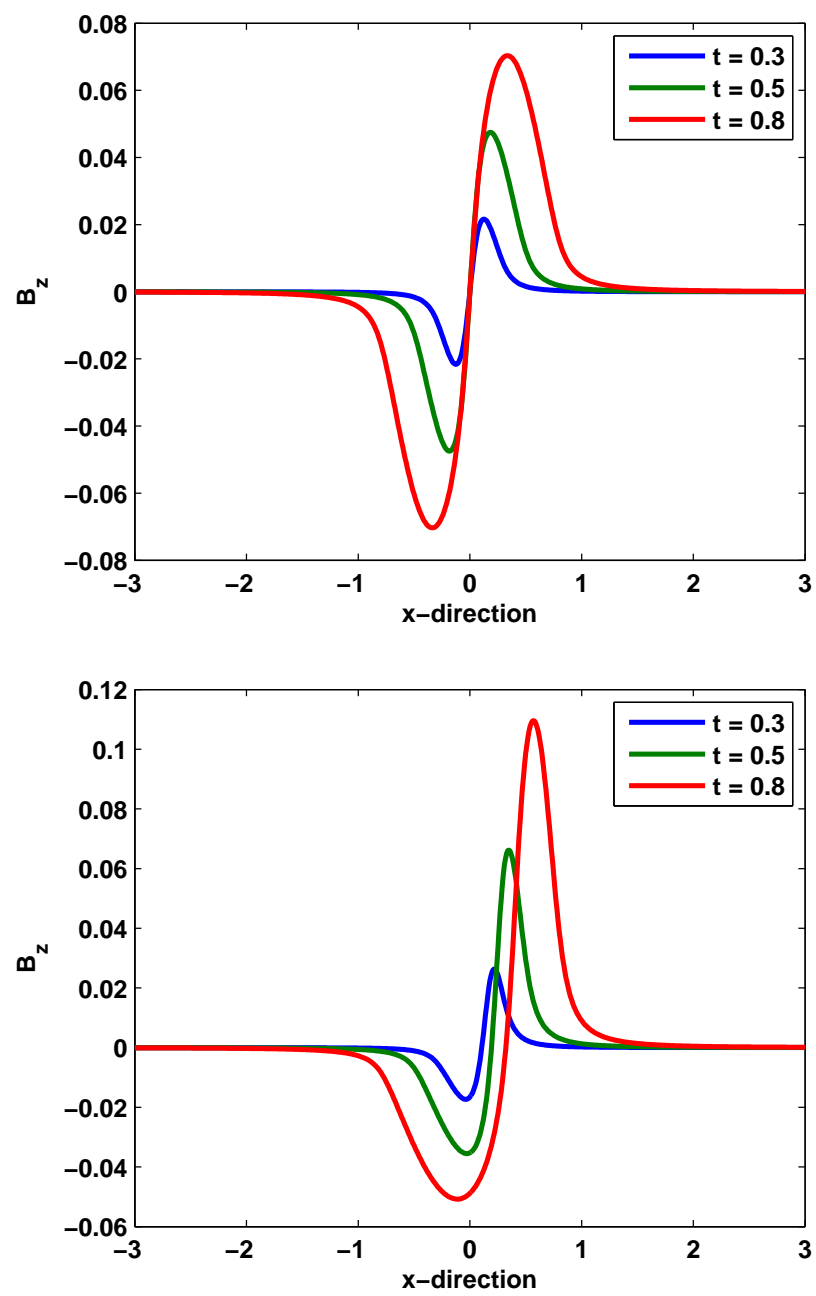

Fig. 7. Behavior of the z-component of the magnetic field in the field reversal region for a magnetic field topology as illustrated in Fig. 1. In the upper panel, the assumption $U=0$ holds, whereas in the lower panel the case $U=0.5 v_{A}$ is shown. Both functions are calculated for times $t=0.3, t=0.5, t=0.8$.

$(0<t \leq 1)$. It can be seen that the magnetic field strength increases with time continuously in both shock structures and that a clear bipolar behavior is exhibited. This is due to the fact that the reconnected magnetic field in the rightward propagating outflow region is oppositely directed to the field in the leftward propagating structure. The lower panel of Fig. 7 shows the magnetic field behavior in the field reversal region for an unsteady behavior of the $\mathrm{X}$-line. A motion with $U=0.5 v_{A}$ in positive $\mathrm{x}$-direction is assumed. The magnetic field in the leftward propagating shock structure has finite values over a larger $\mathrm{x}$-range, due to the size of the shock structure that is stretched in $\mathrm{x}$-direction. The field strength does not reach values as high as in the situation for $U=0$. An opposite situation is given in the rightward plasma bulge, due to a compression of the outflow region in $\mathrm{x}$-direction. In the latter, the magnetic field strength is approximately doubled compared to the field strength in the leftward propagating structure for $t=0.8$.

\section{Magnetic field behavior in the inflow region}

The magnetic field in the outflow region from Eq. (6) yields with Eq. (2),

$\mathbf{B}=\left(\epsilon B_{x}^{(1)},-\epsilon \frac{1}{U \mp 1} E_{r}\left(\frac{x \mp t}{U \mp 1}\right)\right)$.

An approach for the magnetic field in the inflow region can be achieved by using a Taylor expansion (1) as,

$\mathbf{B}=\left(1+\epsilon B_{x}{ }^{(1)}, \epsilon B_{z}^{(1)}\right)$.

Using the jump relation $\llbracket B_{n} \rrbracket=0$ and $\mathbf{n}=\left(\epsilon \frac{\partial f}{\partial x},-1\right)$, gives

$$
\begin{aligned}
& \epsilon \frac{\partial f}{\partial x}+\left.\epsilon^{2} B_{x}{ }^{(1)}\right|_{z=0} \frac{\partial f}{\partial x}-\left.\epsilon B_{z}{ }^{(1)}\right|_{z=0}=\left.\epsilon^{2} B_{x}{ }^{(1)}\right|_{z=0} \frac{\partial f}{\partial x}+ \\
& +\epsilon \frac{1}{U \mp 1} E_{r}\left(\frac{x \mp t}{U \mp 1}\right) .
\end{aligned}
$$

Neglecting terms of order $\mathcal{O}\left(\epsilon^{2}\right)$, the boundary condition $\left.B_{z}{ }^{(1)}\right|_{z=0}$ in the inflow region can be written as

$\left.B_{z}^{(1)}\right|_{z=0}=-\frac{1}{U \mp 1} E_{r}\left(\frac{x \mp t}{U \mp 1}\right)+\frac{\partial f}{\partial x}$.

The shape of the shock from Eq. (4) appears in the normalized case as

$f^{ \pm}(x, t)= \pm \frac{1}{(1 \mp U)^{2}}(x-U t) E_{r}\left(\frac{x \mp t}{U \mp 1}\right)$.

Hence, the first order component $B_{z}$ at $z=0$ in the inflow region is

$$
\begin{aligned}
& \left.B_{z}{ }^{(1)}\right|_{z=0}=-\frac{1}{U \mp 1} E_{r}\left(\frac{x \mp t}{U \mp 1}\right) \pm \frac{1}{(1 \mp U)^{2}} \times \\
& \times\left(E_{r}\left(\frac{x \mp t}{U \mp 1}\right)+\frac{x-U t}{U \mp 1} E_{r}{ }^{\prime}\left(\frac{x \mp t}{U \mp 1}\right)\right),
\end{aligned}
$$

where $E_{r}{ }^{\prime}(x \mp t / U \mp 1)$ is the derivative of the electric field with respect to $x$. With the solution of the Dirichlet problem in a half plane, given by the Poisson integral (Spiegel, 1964), we can compute the behavior of the magnetic field in the inflow region, using

$B_{x}^{(1)}=\frac{1}{\pi} \int_{-t}^{t} \frac{(x-\xi) B_{z}^{(1)}(\xi, 0, t)}{(x-\xi)^{2}+z^{2}} d \xi$,

and

$B_{z}^{(1)}=\frac{z}{\pi} \int_{-t}^{t} \frac{B_{z}^{(1)}(\xi, 0, t)}{(x-\xi)^{2}+z^{2}} d \xi$,

where $B_{z}{ }^{(1)}(\xi, 0, t)$ is the magnetic field z-component varying along the $\mathrm{x}$-axis as in Eq. (9). The total magnetic field 

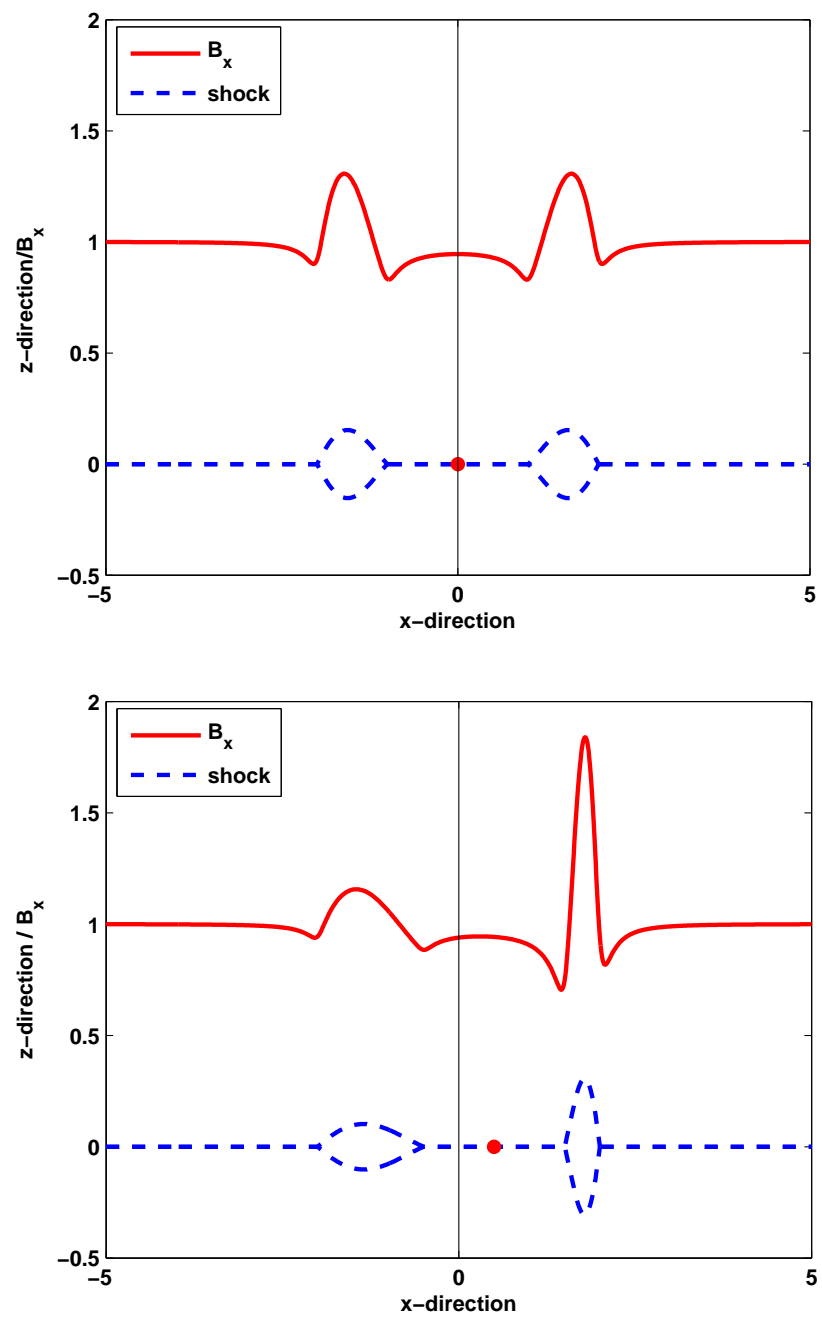

Fig. 8. Behavior of the magnetic field $x$-component in the inflow region. In the upper panel, the assumption $U=0$ holds, whereas in the lower panel, the case $U=0.5 v_{A}$ is shown. Both functions are calculated for $t=2$. Besides the magnetic field strength, the actual positions of the field reversal regions are shown. The dot represents the final position of the reconnection line again. $B_{x}$ is blown up by the factor 10 for a better visibility.

in the inflow region can be achieved by using Eqs. (10), (11) and (7). Figures 8 and 9 show the behavior of the $x-$ and $\mathrm{z}$-components of the magnetic field in the inflow region.

The upper panel of Fig. 8 shows the behavior of the magnetic field $\mathrm{x}$-component in the case of a steady-state X-line for a point in time during switch-off phase. The actual positions of the shocks are displayed as well. Discussing the behavior of the magnetic field from left to right, the first signature is a decrease in the field strength. This is due to an increase in the magnetic field z-component, resulting from a change in the magnetic field topology due to the appearance of the shock. At the location, where the shock reaches its highest elongation in z-direction, the magnetic field strength
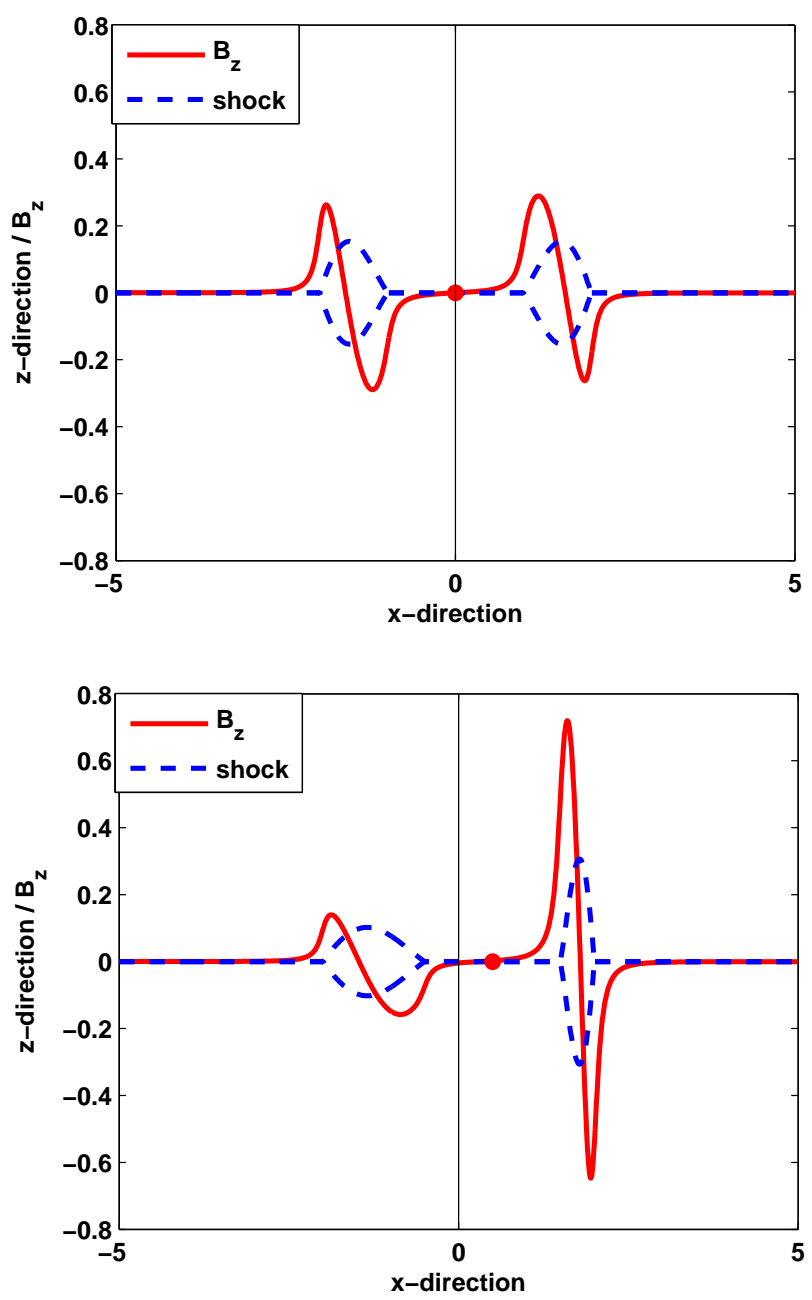

Fig. 9. Behavior of the magnetic field z-component in the inflow region. In the upper panel, the assumption $U=0$ holds, whereas in the lower panel, the case $U=0.5 v_{A}$ is shown. Both functions are calculated for $t=2$. The change in the magnetic field strength as well as the actual positions of the field reversal regions are shown. The dot represents the final position of the reconnection line again. $B_{z}$ is blown up by the factor 10 for a better visibility.

in x-direction is maximized. The lower panel shows the situation for the case of a moving X-line with $U=0.5 v_{A}$. It can be seen that the maximum field strength for the leftward propagating shock is smaller than in the case of $U=0$, due to a smaller elongation of the shock in z-direction. For the shock moving rightward, the situation is vice versa, since the rightward shock is extended in z-direction. Thus, the magnetic field strength is enhanced.

Figure 9 shows the same situation, but displays the magnetic field z-component in the inflow region. In the inflow region, we assume a purely $x$ directed magnetic field before reconnection is initiated. Thus, the field strength in $\mathrm{z}-$ direction is zero without the appearance of the shock structures. In the vicinity of the shocks, a bipolar structure can 

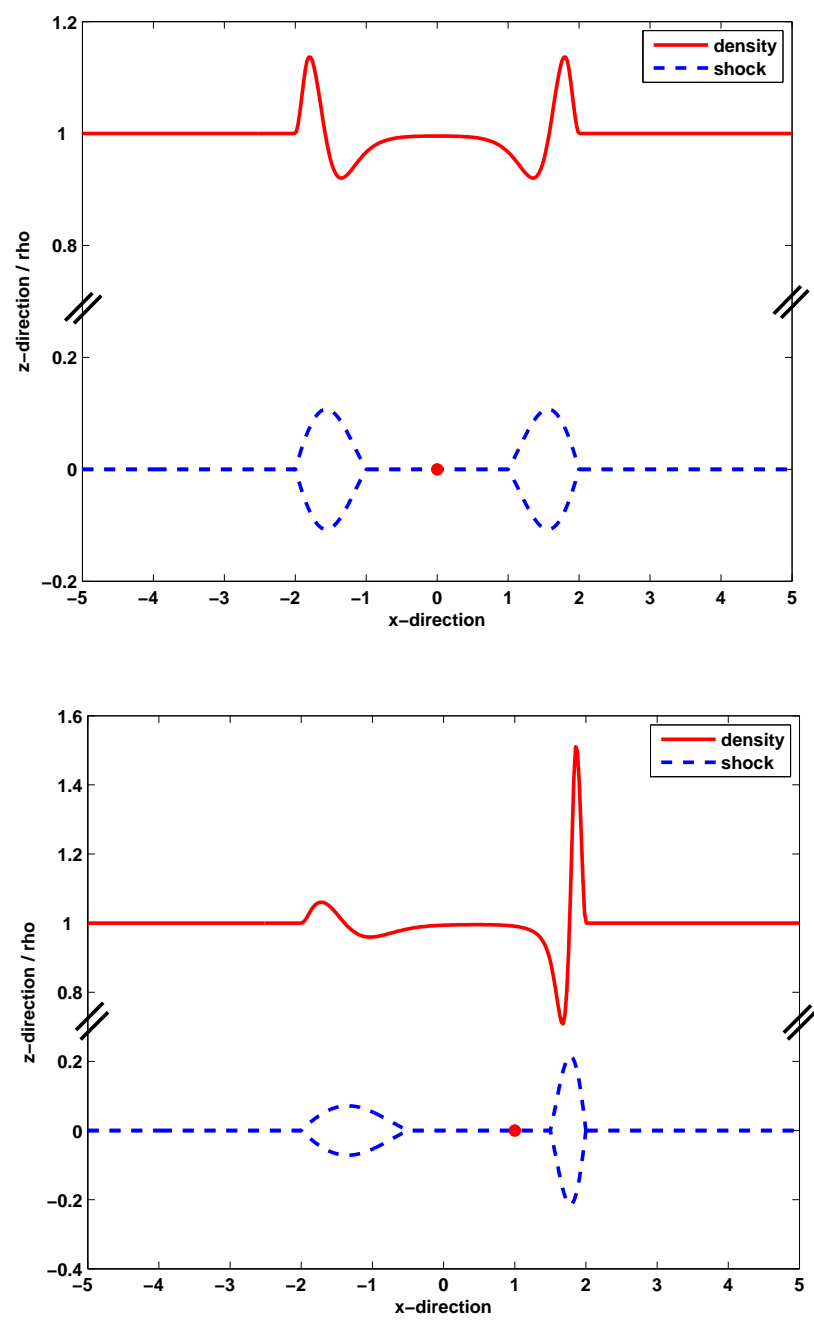

Fig. 10. Density perturbations and corresponding shocks for $U=0$ (upper panel) and $U=0.5$ (lower panel) and $z=0.5$. The calculations were done for $\beta=1$. The compression factor for the shocks is $\rho_{0} / \rho_{\mathrm{FRR}}=0.7$.

be observed. With the appearance of the shock, the magnetic field is forced to develop a magnetic field z-component, which drops to zero in the vicinity of the peak of the shock, where the magnetic field again is purely $x$ directed. In the lower panel, the situation is shown for the case $U=0.5 v_{A}$. Around the leftward propagating shock structure, the magnetic field is less enhanced than in the case of $U=0$, whereas the maximum magnetic field strength in z-direction around the rightward shock is increased.

\section{Effects of compressibility}

In the compressible case, disturbances can not be seen as potentials and they can not be computed with the aid of Poisson integrals. Therefore, we work with the Cagniard-deHoop method for the compressible case (Semenov et al., 2005a).

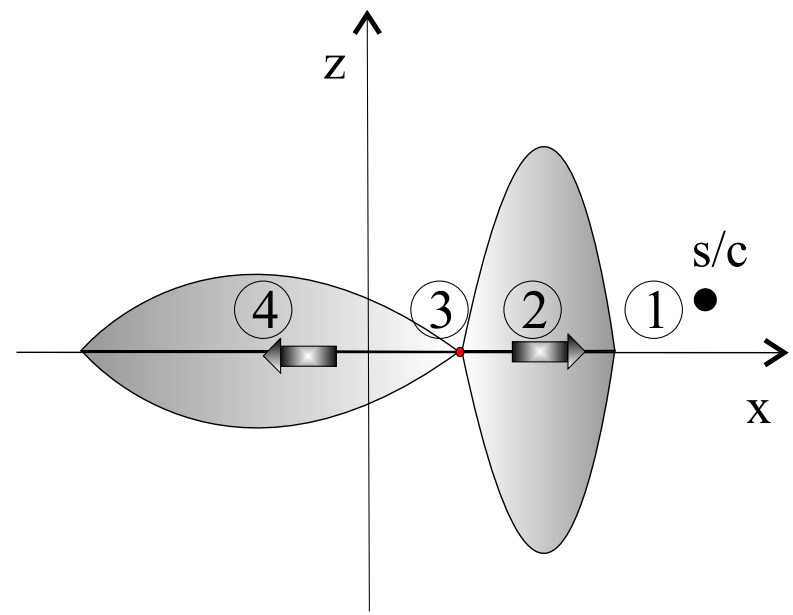

Fig. 11. Spacecraft in the vicinity (region 1) of a reconnection event. The shaded regions denote the outflow regions and the arrows show the direction of the accelerated plasma. The X-line moves rightwards. In the course of time, the spacecraft gets passed by regions $1-4$, measuring different plasma and magnetic field conditions in each region.

For this case, we accomplished a frame transformation to the rest-frame of the reconnection line. In this case, the configuration is given by a resting $\mathrm{X}$-line and a plasma flow with velocity $-U$, which leads to the same asymmetries as mentioned in the previous sections. The compression, and therefore the shape of the shocks, depends on the plasma beta and the adiabatic index $\gamma$,

$\rho_{\mathrm{FRR}}=\rho_{0} \frac{\gamma(\beta+1)}{\gamma(\beta+1)-1}$,

where $\rho_{\text {FRR }}$ and $\rho_{0}$ correspond to the plasma density in the field reversal (outflow) region and inflow region, respectively. The density profiles for different velocities of the reconnection line are shown in Fig. 10. Whereas density is assumed to be constant in the incompressible case, the density variation in a compressible plasma is rather large. Due to the appearance of a moving obstacle, the plasma is compressed at the leading front and diluted in the wake of the outflow region. The function $\rho(x)$ shows the typical bipolar variation, similar to the magnetic field behavior. This effect is strengthened for a moving X-line. The decay of the reconnection layer shows the appearance of switch-off shocks also in the compressible case. The effects of compressibility on the outflow regions result in the expected compression of the regions, which leads to a smaller maximum and minimum of the perturbations (see Semenov et al., 2005b).

\section{Qualitative observational aspects}

Let us consider a spacecraft positioned as in Fig. 11. The $\mathrm{X}$-line propagates rightwards with sub-Alfvénic velocity $U$. 
Eventually, the rightward propagating plasma bulge (region 2) reaches the spacecraft, which measures considerable plasma flow and a magnetic field z-component. After region 2 passed by, the spacecraft is located in the intermediate region between the two shock structures (region 3 ). If reconnection is still in progress, the shocks are not detached from the $\mathrm{X}$-line and also the leftward propagating plasma bulge (region 4) reaches the spacecraft, which detects again plasma flow and a magnetic field z-component, both oppositely directed as in region 2. In magnetotail data this event can be observed as a flow reversal (e.g., Deng et al., 2005). Since the plasma in region 4 moves with local Alfvén velocity towards the left, but the entire shock structure together with the X-line with sub-Alfvénic speed towards the right, the spacecraft is "trapped" in region 4 until reconnection ceases. When this happens, the shock structures detach from the initial X-line and after some time the spacecraft finds itself in region 3 again. Thus, it is not possible that both plasma structures entirely pass a spacecraft, located rightwards from the reconnection scene.

\section{Summary}

We extended the time-dependent Petschek-type reconnection model for the situation of a moving reconnection line, focusing on the discussion of the shock structures, which enclose the outflow regions, and the behavior of the magnetic field in the inflow and outflow regions. It is shown that the shock structures on the left-hand and right-hand side of the diffusion region lose their symmetric behavior under the assumption of an unsteady X-line behavior. By modelling a rightward (in positive $\mathrm{x}$-direction) moving $\mathrm{X}$-line situation, the shock structure, which evolves on the right-hand side, is squeezed in $\mathrm{x}$-direction and elongated in z-direction. For the shock structure propagating in the opposite direction, the situation is vice versa. The degree of asymmetry depends on the velocity of the $\mathrm{X}$-line and increases with the velocity. The classical time-dependent Petschek solution appears as a border case for the situation of a steady-state reconnection line, with velocity $U=0$. The behavior of the magnetic field was investigated for the z-component in the outflow region, since an $x$-component appears only in form of disturbances. The typical bipolar behavior of the magnetic field z-component is extended by an asymmetry, appearing in the outflow regions left- and rightwards to the (initial) diffusion region. In the outflow region, propagating in the same direction as the $\mathrm{X}$-line, the field strength is enhanced. The behavior of the magnetic field in the inflow region is evaluated for $\mathrm{x}$ - and z-components as a comparison of steady-state and unsteady $\mathrm{X}$-line behavior, featuring asymmetric structures for a moving X-line assumption. Around the shock structures propagating in the same direction as the $\mathrm{X}$-line, the magnetic field $\mathrm{X}$ - and z-components are enhanced. Finally, this paper delivers an appropriate theoretical model, describing the effects of a moving reconnection line, and can be seen as a basis for future concrete applications.

Acknowledgements. This work is supported by the "Verwaltungsstelle für Auslandsbeziehungen" of the Austrian Academy of Sciences, the Austrian "Fonds zur Förderung der wissenschaftlichen Forschung" under grant P17099-N08 and the "Österreichischen Austauschdienst" under project No. I.12/04. Also acknowledged is support by the Russian Academy od Sciences, RFBR grant No. 04-05-64935.

Topical Editor I. A. Daglis thanks S. W. H. Cowley and another referee for their help in evaluating this paper.

\section{References}

Akhiezer, A. I., Akhiezer, I. A., Polovin, R. V., Sitenko, A. G., and Stepanov, K. N.: Plasma Electrodynamics, Pergamon Press, Oxford - New York - Toronto - Syndey - Braunschweig, 1975.

Alexeev, I. V., Semenov, V. S., and Biernat, H. K.: First-order effects in time-dependent Petschek-type reconnection, J. Plasma Phys., 30, 1-14, 2001.

Baker, D. N., Peterson, W. K., Eriksson, S., Li, X., Blake, J. B., Burch, J. L., Daly, P. W., Dunlop, M. W., Korth, A., Donovan, E., Friedel, R., Fritz, T. A., Frey, H. U., Mende, S. B., Roeder, J., and Singer, H. J.: Timing of magnetic reconnection initiation during a global magnetospheric substorm onset, Geophys. Res. Lett., 29, 2190, doi:10.1029/2002GL015539, 2002.

Baumjohann, W. and Treumann, R. A.: Basic Plasma Physics, Imperial College Press, London, 1996.

Biernat, H. K., Heyn, M. F., and Semenov, V. S.: Unsteady Petschek reconnection, J. Geophys. Res., 92, 3392-3396, 1987.

Cattell, C., Dombeck, J., Wygant, J., Drake, J. F., Swisdak, M., Goldstein, M. L., Keith, W., Fazakerely, A., André, M., Lucek, E., and Balogh, A.: Cluster observations of electron holes in association with magnetotail reconnection and comparison to simulations, J. Geophys. Res., 110, A01211, doi:10.1029/2004JA010519, 2005.

Deng, X. H., Tang, R. X., Nakamura, R., Baumjohann, W., Zhang, T. L., Daly, P. W., Rème, H., Carr, C. M., Balogh, A., Liu, Z. X., and Wang, J. F.: Observation of reconnection pulses by Cluster and Double Star, Ann. Geophys., 23, 2921-2927, 2005, http://www.ann-geophys.net/23/2921/2005/.

Heyn, M. F., Biernat, H. K., Rijnbeek, R. P., and Semenov, V. S.: The structure of reconnections layers, J. Plasma Phys., 40, 235252, 1988.

Kiendl, M. T., Semenov, V. S., Kubyshkin, I. V., Biernat, H. K., Rijnbeek, R. P., and Besser, B. P.: MHD analysis of Petschek-type reconnection in non-uniform field and flow geometries, Space Sci. Rev., 79, 709-755, 1997.

Owen, C. J. and Cowley, S. W. H.: Simple models of timedependent reconnection in a collision-free plasma with an application to substorms in the geomagnetic tail, Planet. Space Sci., 35, 451-466, 1987.

Petschek, H. E.: Magnetic field annihilation, in: Physics of Solar Flares, edited by: Hess, W. N., NASA Spec. Publ. 50, 425-439, 1964.

Pudovkin, M. I. and Semenov, V. S.: Magnetic field reconnection theory and the solar wind-magnetosphere interaction: A review, Space Sci. Rev., 41, 1-89, 1985. 
Rijnbeek, R. P., Semenov, V. S., Shmalts, S. S., Biernat, H. K., Heyn, M. F., and Besser, B. P.: Time-dependent reconnection in a current sheet with velocity sheer, Planet. Space Sci. 39, 13771395, 1991.

Rijnbeek, R. P. and Semenov, V. S.: Features of a Petschek-type reconnection model, Trends in Geophys. Res., 2, 247-268, 1993.

Runov, A., Nakamura, R., Baumjohann, W., Treumann, R. A., Zhang, T. L., Volwerk, M., Vörös, Z., Balogh, A., Glaßmeier, K.H., Klecker, B., Rème, H., and Kistler, L., Current sheet structure near magnetic X-line observed by Cluster, Geophys. Res. Lett., 30, 1579, doi:10.1029/2002GL016730, 2003.

Semenov, V. S., Kubyshkin, I. V., Lebedeva, V. V., Sidneva, M. V., Biernat, H. K., Heyn, M. F., Besser, B. P., and Rijnbeek, R. P., Time-dependent localized reconnection of skewed magnetic fields, J. Geophys. Res. 97, 4251-4263, 1992.

Semenov, V. S., Kubyshkin, I. V., Rijnbeek, R. P., and Biernat, H. K.: Analytical theory of unsteady Petschek-type reconnection, in: Physics of magnetic reconnection in high-temperature plasmas, edited by: Ugai, M., Research Signpost, Trivandrum, India, 35-68, 2004.
Semenov, V. S., Penz, T., Heyn, M. F., Ivanov, I. B., Kubyshkin, I. V., Biernat, H. K., and Ivanova, V. V.: Reconstruction of the reconnection rate from magnetic field disturbances in an incompressible plasma, in: Solar-planetary relations, edited by: Biernat, H. K., Lammer, H., Vogl, D. F., and Mühlbachler, S., Research Signpost, Trivandrum, India, 261-302, 2005a.

Semenov, V. S., Penz, T., Ivanova V. V., Sergeev, V. A., Biernat, H. K., Nakamura, R., Heyn, M. F., Kubyshkin, I. V., and Ivanov, I. B.: Reconstruction of the reconnection rate from Cluster measurements: First results, J. Geophys. Res., 110, A11217, doi:10.1029/2005JA011181, 2005b.

Sonnerup, B. U. O.: Magnetic field reconnection in highly conducting incompressible fluid, J. Plasma Physics, 4, 161-174, 1970.

Spiegel, M. R.: Complex Variables, Schaum's Outline, McGrawHill, London, 1964. 